Erratum

\title{
Erratum to "N-Screen Aware Multicriteria Hybrid Recommender System Using Weight Based Subspace Clustering"
}

\author{
Farman Ullah, Ghulam Sarwar, and Sungchang Lee \\ Department of Information \& Communication, Korea Aerospace University, Goyang 412-791, Republic of Korea \\ Correspondence should be addressed to Sungchang Lee; sclee@kau.ac.kr \\ Received 21 May 2015; Accepted 3 June 2015 \\ Copyright (c) 2015 Farman Ullah et al. This is an open access article distributed under the Creative Commons Attribution License, \\ which permits unrestricted use, distribution, and reproduction in any medium, provided the original work is properly cited.
}

In the published paper titled "N-Screen Aware Multicriteria Hybrid Recommender System Using Weight Based Subspace Clustering," in Figure 2, it was not "Device pynamic profile," it is "Device dynamic profile" and it was not "inforomation" it is "information." Also, in Algorithm 2, there was an issue with square root placement in Equation (ii). The right equation is as follows:

$$
\begin{aligned}
& \text { usersim }\left(R_{t}, R_{c}\right) \\
& =\frac{\sum_{g}^{G} \sum_{n=1}^{N} w_{g} v_{n} R_{t(g, n)} R_{c(g, n)}}{\sqrt{\sum_{g}^{G} \sum_{n=1}^{N}\left(w_{g} v_{n} R_{t(g, n)}\right)^{2} \sum_{g}^{G} \sum_{n=1}^{N}\left(w_{g} v_{n} R_{c(g, n)}\right)^{2}}} .
\end{aligned}
$$




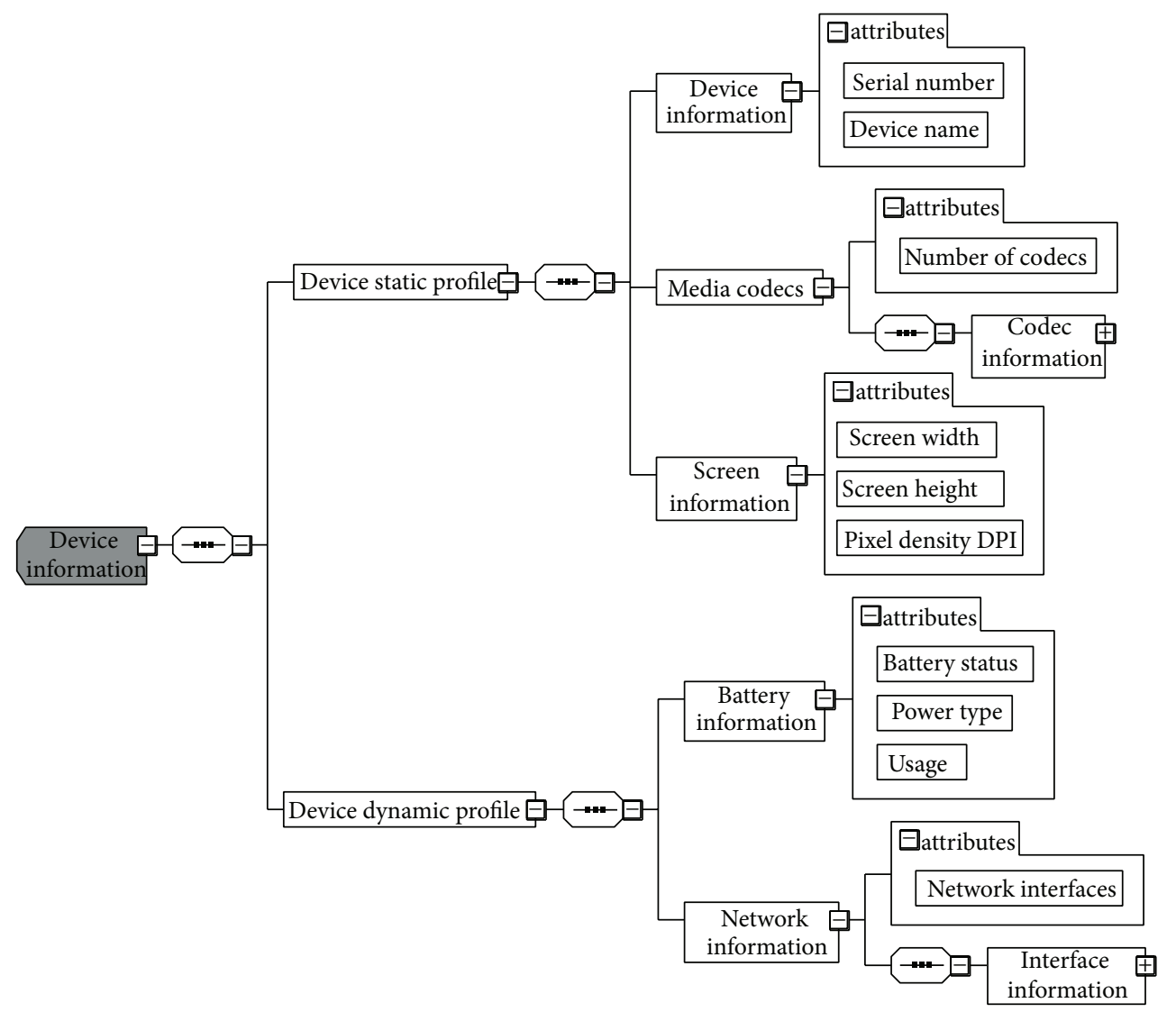

FIGURE 2: Schema for user N-screen device profile attributes. 

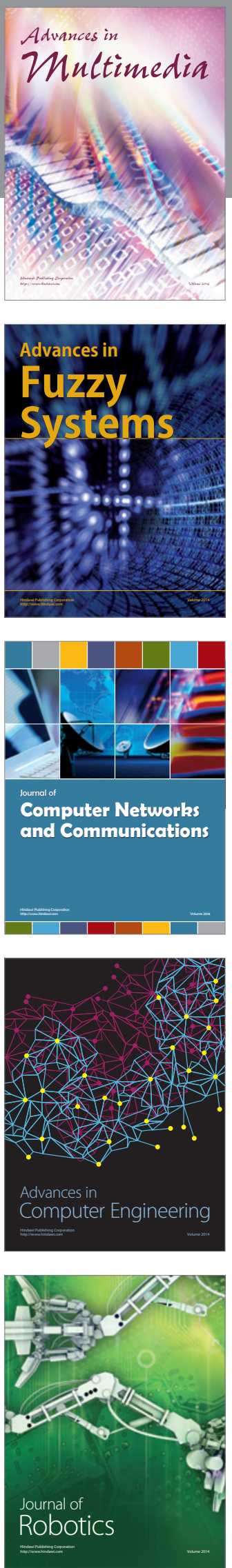

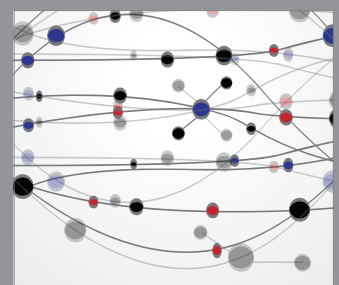

The Scientific World Journal
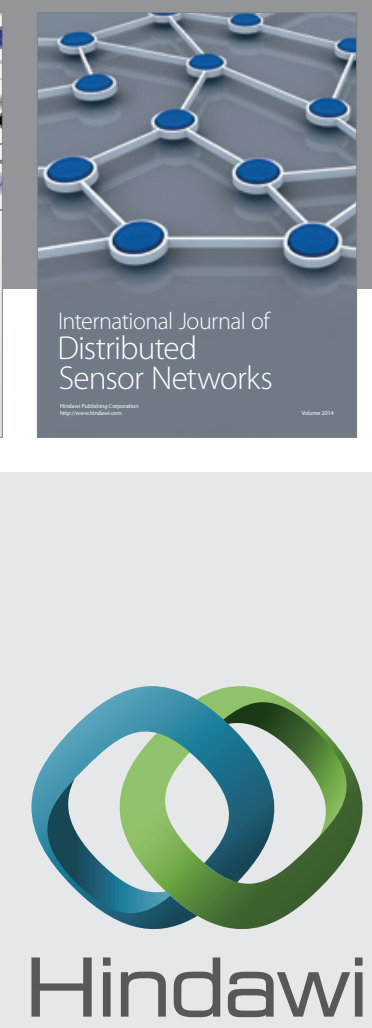

Submit your manuscripts at

http://www.hindawi.com
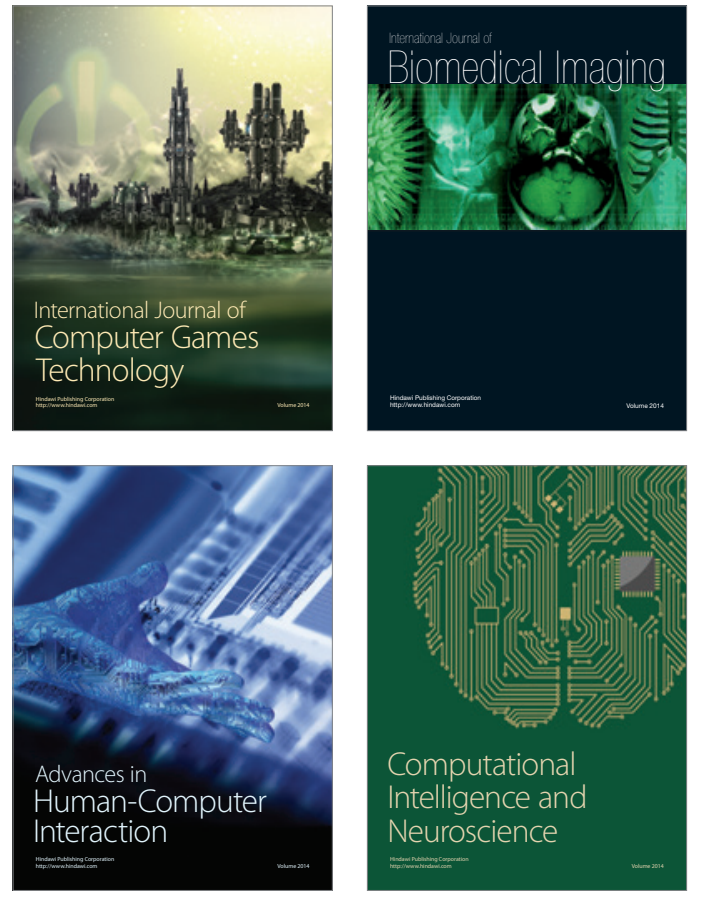
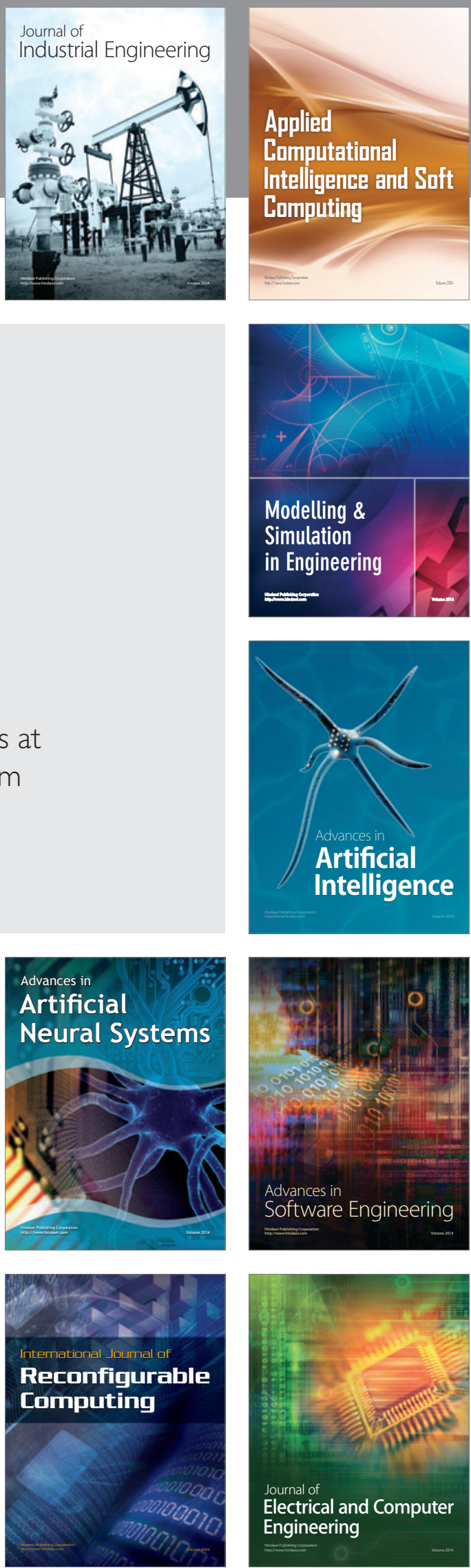